\title{
Addressing partial identification in climate modeling and policy analysis
}

\author{
Charles F. Manski ${ }^{a, 1}{ }^{\circ}$, Alan H. Sanstad ${ }^{b}$, and Stephen J. DeCanio ${ }^{c, 2}([)$ \\ ${ }^{a}$ Department of Economics and Institute for Policy Research, Northwestern University, Evanston, IL 60208; ${ }^{\circ}$ Private address, Berkeley, CA 94709; \\ and 'Department of Economics, University of California, Santa Barbara, CA 93106
}

Contributed by Charles F. Manski, December 30, 2020 (sent for review November 2, 2020; reviewed by Robert Pindyck and Robert Rosner)

Numerical simulations of the global climate system provide inputs to integrated assessment modeling for estimating the impacts of greenhouse gas mitigation and other policies to address global climate change. While essential tools for this purpose, computational climate models are subject to considerable uncertainty, including intermodel "structural" uncertainty. Structural uncertainty analysis has emphasized simple or weighted averaging of the outputs of multimodel ensembles, sometimes with subjective Bayesian assignment of probabilities across models. However, choosing appropriate weights is problematic. To use climate simulations in integrated assessment, we propose, instead, framing climate model uncertainty as a problem of partial identification, or "deep" uncertainty. This terminology refers to situations in which the underlying mechanisms, dynamics, or laws governing a system are not completely known and cannot be credibly modeled definitively even in the absence of data limitations in a statistical sense. We propose the min-max regret (MMR) decision criterion to account for deep climate uncertainty in integrated assessment without weighting climate model forecasts. We develop a theoretical framework for cost-benefit analysis of climate policy based on MMR, and apply it computationally with a simple integrated assessment model. We suggest avenues for further research.

climate policy $\mid$ climate modeling $\mid$ structural uncertainty $\mid$ partial identification | decision-making

omputational modeling of the global climate system provides a foundation for estimating the effects of greenhouse gas (GHG) mitigation and other policies on global climate change. Climate models project the future path of the climate as a function of trajectories of anthropogenic GHG emissions and other inputs. * They are used to predict the climatic and other impacts of altering emission trajectories by means of various GHG reduction policies, which, in turn, supports analysis of how to address the climate change problem.

Climate models aim to represent the immensely complicated climate system in a tractable manner. Scientific understanding of the climate, as developed over decades of research by many scholars around the world, is, at this point, remarkable in its scope and depth. It remains, however, incomplete, and continues to evolve. Hence, the construction and use of climate models entail choices by modelers regarding model elements that are not fully specified by physical theory or exactly determined by empirical evidence. Over time, different sets of choices have yielded different climate models

Researchers have sought to understand and quantify the uncertainties in climate models and the predictions that they yield. One primary approach is multimodel ensemble (MME) analysis of intermodel structural choices (1). This paper offers a perspective on the uncertainties MME analysis seeks to address, from the vantage point of econometrics, decision theory, and energy policy analysis. We frame climate model uncertainty as a problem of partial identification, or "deep uncertainty." This terminology refers to situations in which the underlying mechanisms governing a system are not completely known and cannot be credibly modeled definitively even in the absence of data limitations in a statistical sense.

The predictions yielded by a partially identified model of a system are set-valued rather than exact or probabilistic. Thus, partial identification generates what is referred to in various disciplines as "ambiguity," "model uncertainty," "deep uncertainty," or "epistemic uncertainty." Study of partial identification has origins mainly in econometrics, which applies statistical methods to study economic phenomena. In this field, partial identification refers to circumstances in which the probability distribution governing a stochastic system is not completely known theoretically and its unknown aspects cannot be fully learned empirically even in the absence of data limitations in the sense of statistical imprecision. In the limit case where these unknown aspects can be fully learned empirically given sufficient data, the probability distribution is said to be point identified or simply identified.

The fundamental mathematical and physical theory underlying computational climate modeling is not based on probability distributions but rather comprises a set of deterministic nonlinear partial differential equations describing large-scale atmospheric dynamics. However, due to incomplete knowledge of other physical processes and differences in ways to represent them numerically, multiple climate models have been developed around the world and are currently in use, each reflecting different but credible choices in model design and implementation. Existing models yield different projections of the global climate

\section{Significance}

Incomplete scientific understanding of the climate and challenges in numerical computation have resulted in numerous climate simulation models being developed and used to generate ensembles, or sets, of climate projections as functions of single greenhouse gas (GHG) emissions scenarios. We propose a "deep uncertainty" approach to framing and analyzing these ensemble model outputs that allows their use in climaterelated decision-making without reliance on problematic model weighting schemes. We provide a theoretical framework and an illustrative numerical model to show how single, nearly optimal decisions can be made regarding GHG abatement without requiring single, average climate model projections.

Author contributions: C.F.M. and A.H.S. designed research; C.F.M., A.H.S., and S.J.D. performed research; C.F.M., A.H.S., and S.J.D. contributed new reagents/analytic tools; C.F.M., A.H.S., and S.J.D. analyzed data; and C.F.M., A.H.S., and S.J.D. wrote the paper.

Reviewers: R.P., Massachusetts Institute of Technology; and R.R., University of Chicago.

The authors declare no competing interest.

Published under the PNAS license.

${ }^{1}$ To whom correspondence may be addressed. Email: cfmanski@northwestern.edu.

${ }^{2}$ Emeritus.

This article contains supporting information online at https://www.pnas.org/lookup/suppl/ doi:10.1073/pnas.2022886118/-/DCSupplemental.

Published April 7, 2021

*This paper focuses on coupled atmospheric, ocean, and land surface models; we follow the custom of using the term "climate model" to refer to these coupled systems. 
even with broadly harmonized input assumptions. Put differently, neither a "consensus" climate model nor quantitative climate projections can be definitively specified with current knowledge. These intermodel differences are referred to as reflecting "structural" uncertainty. We show how this can be framed in terms of partial identification or deep uncertainty.

This paper applies partial-identification thinking to climate modeling in two ways. First, we argue on decision-theoretic grounds against the prevalent practice in climate modeling of computing simple or weighted averages of predictions across models considered in MME analysis. Computation of simple averages of predictions assumes that equal weight should be given to each model, an assumption lacking a compelling foundation (2). But determining appropriate weights is challenging, for reasons including the weak link between metrics of model validity and predictive skill (1). Some researchers have attempted to motivate computation of weighted averages as an exercise in Bayesian judgment, the weights expressing subjective probabilities that particular models represent truth (3). However, it may be difficult to judge the credibility of the subjective probability distribution used. Rather than compute averages of, or assign probability distributions to, predictions across models, partial identification analysis would present the set of predictions yielded by model ensembles as the starting point for decisionmaking, foregoing an attempt to reduce the set to a point through averaging.

Second, we use classical ideas in decision theory to show how these ensemble outputs can be applied directly in policy applications of climate modeling, specifically the min-max (4) and min-max regret (MMR) (5) decision rules. In decision problems in general, min-max reflects the principle of preparing for the worst case. In the climate context, it would entail making policy decisions, such as carbon abatement levels, using the most "pessimistic" climate model in an ensemble-the one projecting the highest temperature changes. MMR uses multimodel information in a more nuanced way. If a climate policy maker selects one model from an ensemble and chooses, say, an emissions abatement path that is optimal for that model according to some economic metric, the economic penalty, or regret, is the excess cost of that abatement path if a different model is actually the correct one. That is, regret measures the potential suboptimality of policies relative to climate models. The MMR rule is to choose a policy that minimizes the maximum regret, or largest degree of suboptimality, across the climate model ensemble. ${ }^{\dagger}$

MMR has been applied in several policy areas, including criminal justice (6) and public health (7). It has been used in computational modeling of energy and climate policy in a number of studies (8-12). [Cai (13) reviews this research.] The present paper complements this previous work in several ways. The paper is aimed, in part, at climate modelers and other researchers who are nonspecialists in economics but interested in the policy applications of climate modeling. Our approach is primarily conceptual rather than computational. We emphasize the partial identification paradigm and its suitability for framing structural uncertainty in climate models. We provide a theoretical discussion of MMR for climate policy, to highlight the basic idea in noncomputational form. We then present a deliberately simple numerical integrated assessment (IA) example of MMR applied to carbon abatement policy, in order to illustrate the method in a transparent way.

In what follows, Partial Identification in Econometrics and Climate Modeling discusses the theory of partial identification as

\footnotetext{
${ }^{\dagger}$ The other primary approach to studying climate model uncertainty is Perturbed Physics Ensemble (PPE) analysis of parametric uncertainty. Mutatis mutandis, the concepts and methods discussed in this paper can also be applied to understanding PPEs from a decision perspective.
}

developed in econometrics and describes how climate model uncertainties can be framed in this way. Partial Identification and $M M E$ Analysis discusses technical aspects of structural uncertainties in climate modeling as a partial identification problem. Climate Policy under Partial Identification turns to decisionmaking under partial identification, providing background and discussing the min-max and MMR decision rules. A Numerical Example develops a numerical example illustrating their application to the problem of choosing dynamic carbon abatement policies that optimally balance abatement costs and climate economic damages. The paper ends with a summary and concluding remarks.

\section{Partial Identification in Econometrics and Climate Modeling}

Econometrics is the term used by economists to describe study of the logic of empirical inference. Whatever the field of science may be, the logic of empirical inference is summarized by the following relationship: assumptions + data $\rightarrow$ conclusions. Data alone do not suffice to draw useful conclusions. Inference requires assumptions that relate the data to a question of interest. Holding fixed the available data, a fundamental difficulty of empirical research is deciding what assumptions to maintain.

Identification and Statistical Inference. Economists and many other researchers use sample data to learn about theoretically unknown aspects of the mechanisms that govern stochastic systems. Empirical research confronts problems of statistical imprecision and identification problems. Statistical theory characterizes the inferences that can be drawn by observing a finite sample of outcomes generated by a system. Identification analysis studies inferential difficulties that persist even when sample size grows without bound. The distinction between identification and statistical inference, first formalized by Koopmans (14), has long been central to the field of econometrics.

A simple example of statistical imprecision occurs when one observes a random sample of outcomes generated by a stationary system and uses this sample average to estimate the mean outcome that the system produces. Statisticians typically measure imprecision of the estimate by its variance, which decreases to zero as sample size increases. The famous "Laws of Large Numbers" imply that imprecision vanishes as sample size increases to infinity.

Identification problems encompass the spectrum of issues that are sometimes called nonsampling errors or data-quality problems. These issues cannot be resolved by amassing so-called "big data." They may be mitigated by collecting better data, but not by merely collecting more data.

A simple example of an identification problem is generated by measurement errors. Suppose that a stationary system generates a random sample of outcomes, but one observes these outcomes with error. Increasing sample size adds new observations, but it also yields further measurement errors. If one has incomplete knowledge of the process creating measurement errors and cannot collect better data that are error-free, one cannot precisely learn the system mean outcome as sample size increases to infinity.

Point and Partial Identification. For most of the twentieth century, econometricians studying inference commonly thought of identification as a binary event - a parameter of a model is either point identified or it is not. Empirical researchers combined available data with assumptions that yield point identification, and they reported point estimates of model parameters. Economists recognized that point identification often requires strong assumptions that are difficult to motivate, but they saw no other way to perform inference.

Yet there is enormous scope for fruitful inference using weaker and more credible assumptions that partially identify 
objects of interest. A parameter in a probabilistic model is partially identified if the sampling process and maintained assumptions reveal that the object lies in a set, called the identification region or identified set, that is smaller than the logical range of possibilities but larger than a single point. Sample estimates of partially identified objects generically are set-valued.

To illustrate, suppose economic theory posits that there are positive parameters $a, b$, and $c$ such that $Y=(a \cdot c) \cdot X+b+\varepsilon$. Given a random sample of observations of pairs $(X, Y)$, the product $d=a \cdot c$ can be estimated by the method of least squares if it is known that $\varepsilon$ has mean zero conditional on $X$. Statistical imprecision regarding $d$ vanishes as sample size increases. However, absent additional assumptions, all that can be inferred empirically about $a$ and $c$ is that they lie in the set of all pairs of positive real numbers such that $a \cdot c=d$. That is, these parameters are partially identified, with the identification region defined by this identity.

Part of the modern literature on partial identification in econometrics focuses on the identification problems generated by imperfect data quality, including measurement error and missing data. Part focuses on identification of structural econometric models used to describe human behavior and interactions. Manski (15, 16), Tamer (17), and Molinari (18) provide in-depth reviews of the literature at different technical levels and with different emphases. SI Appendix, section 1 provides a brief history and some key references.

Whatever the specific subject under study, a common theme runs through the literature. One first asks what available data combined with relatively weak and highly credible assumptions reveal about the population of interest. One then studies the identifying power of stronger assumptions that still aim to retain acceptable credibility, keeping in mind a principle called (ref. 15, p. 1) the law of decreasing credibility: "The credibility of inference decreases with the strength of the assumptions maintained." This principle implies that researchers face a dilemma as they decide what assumptions to maintain: Stronger assumptions yield inferences that may be more powerful but less credible.

This approach to inference makes clear the conclusions one can draw in empirical research without imposing untenable assumptions. It establishes a domain of consensus among researchers who may hold disparate beliefs about what assumptions are appropriate. Partial identification analysis also makes plain the limits to credible inference. When identification regions turn out to be large, researchers should face up to the fact that the available data and acceptable assumptions do not support inferences as tight as they might like to achieve.

Partial Identification of Climate Models. One might think that econometrics and climate modeling are so different that econometric methodology would have little if any application to climate modeling. In this subsection, we explain why the ideas of partial identification do, in fact, apply to climate models and can serve to frame and interpret their uncertainties.

First, research in both domains faces broadly similar identification problems due to data imperfections. Climate data that might have been available in principle may have measurement errors or be missing in practice, especially data documenting climate dynamics prior to the existence of modern instrumentation. Moreover, climate data in counterfactual settings is unavailable in principle. We cannot observe what climate change would have occurred over the past century if the trajectory of anthropogenic GHG emissions had differed from its actual path.

Second, research in both domains faces the problem of modeling a complex system. The climate system comprises many different physical processes and mechanisms occurring at a range of spatial and temporal scales. Designing and implementing a climate model requires many choices regarding the underlying architecture, the methods of numerical approximation, solution technique, which physical processes to include as model components, and how to represent these processes (19). Climate model structural uncertainty arises from incomplete scientific understanding of physical phenomena, limitations of quality and quantity of empirical data available to fit models, and the need to make approximations for computational tractability.

With respect to underlying scientific principles, all general circulation models of the climate are based on a set of five deterministic equations governing atmospheric variables including temperature, pressure, humidity, and wind flow (20). These socalled "primitive equations" instantiate first-principle laws of physics; they include partial differential equations describing a form of the classical conservation laws of momentum, mass, and energy.

However, the highly complex and nonlinear nature of the climate system makes it inevitable that the computational models used to represent it are subject to a degree of irreducible imprecision (21). Although the basic set of primitive equations is common across models in a theoretical sense, the exact way in which the equations are rendered in a particular model is subject to numerous practical choices of implementation, including choice of coordinate system, discretization approach, and numerical solutions. In addition, climate models differ in the details of which physical processes are selected for inclusion-that is, the overall strategy used to simplify the climate into a tractable form for modeling - and how they are represented. The multiplicity of models and intermodel variations of the quantitative outputs they generate are generally referred to as reflecting "structural" uncertainty (22-25).

It is important to note that this is not an exhaustive description of uncertainty in climate modeling, nor would it be if parametric uncertainty were also taken into account. The factors just discussed are "known unknowns"- they have been explicitly determined to be sources of uncertainty. By contrast, "unknown unknowns" are also presumptively present-it cannot be assumed that the models encompass all physical processes affecting the climate to an extent that is policy relevant.

To summarize, the present state of science, modeling methods, and empirical knowledge is not such that the climate system can be uniquely represented in mathematical or computational form. Nor can climate dynamics be predicted deterministically or stochastically-one can only predict a range of possible trajectories (26). Thus, we see an underlying correspondence between econometric and climate models with respect to structural uncertainty, making the concept of partial identification applicable to climate modeling.

In the following sections, we elaborate on this perspective and show how it can be applied to climate change-related policy decision-making.

\section{Partial Identification and MME Analysis}

At the beginning of the paper, we introduced the terminology of climate model "ensembles." It is difficult to vary a given model's components such as subgrid process representations (much less numerical integration schemes) to assess the effects of variations in structural components on the model's performance. Thus, to quantify and analyze structural uncertainty, sets of models are run to study how the different structural choices across models affect the outputs across the set. This is MME analysis, which we discuss next.

MMEs Background. MMEs are based on model intercomparison projects in which the characteristics and performance of individual and multiple models are analyzed. First conducted in the 1980s (27), such projects have since proliferated and greatly increased in scope and detail. Most MME analyses have been generated in the Coupled Model Intercomparison Project (CMIP), which began in the 1990s and has thus far completed five studies $(28,29)$. These studies examine the individual and collective ability of models to simulate the current and historical 
climate, analyze the factors driving model behavior and intermodel differences, and provide a means of assessing changes in model performance over time (30).

The performance of climate models in simulating present and past climate has been gauged by various metrics based on the mean squared error of differences between model outputs, or "fields," and their observed counterparts, such as global mean temperature and precipitation (31). Comparison of model performance based on such metrics has been conducted using CMIP MMEs of historical simulations (32).

The CMIP and other intercomparison projects have been part of a substantial international research effort to develop and improve the models. Advances in computational hardware and software have also been critical. The models have been refined and improved in a number of ways, including the ability to simulate key aspects of the historical climate.

Nevertheless, there continue to be shortcomings in the models' accuracy and precision (1). There are nontrivial biases and uncertainties in particular fields across models that have persisted through development cycles (33-35). As noted previously, structural uncertainty arises from the fact that theory and evidence do not completely constrain design and implementation choices such as which subgrid processes to include, at what levels of detail. When assessing model performance in historical simulations, there are multiple model fields for which performance metrics can be defined and quantified, and modelers have not been able to develop a single metric that effectively captures all the dimensions of interest (24). Because the relative accuracies of different models can depend on which field is measured, it has not proven possible to unambiguously rank models with respect to performance or to identify a "best" model using historical climate information (31). The extent of structural uncertainty can be gauged by the fact that there are currently several dozen different modeling groups around the world, each running one or more versions of its own model (36).

Abramowitz et al. (37) take the view that different models can be thought of as representing different "working hypotheses" about how to best represent the details of the structure and dynamics of the climate. Parker (26) characterizes the climate modeling community's acceptance of multiple, coexisting models as "model pluralism," with different models seen as complementary. In the language of the present paper, the climate models are only partially identified.

MME Projections of Future Climate. Model pluralism has generally been maintained in MME studies of the future climate, in that the sets of models used in specific studies have usually been determined simply by which modeling teams choose to participate, a procedure Knutti (2) describes as "model democracy." That available models are not assessed or screened for inclusion essentially reflects the unavailability of reliable, agreed-upon criteria for doing so: If ab initio structural uncertainty (partial identification) cannot be resolved through standard evaluation and performance intercomparison, there are no obvious grounds to omit particular models from climate projection studies. Nevertheless, some modelers and climate scientists believe that there are important shortcomings in model democracy for projective MME analysis (2). Developing better approaches has been the focus of considerable research on narrowing model selection criteria and/or finding ways of assessing candidate models according to some informational criterion.

Most of this work is closely tied to, or part of, a broader longterm effort to address uncertainty in MMEs of future climate. A number of methods have been developed and applied to MME uncertainty quantification and analysis, virtually all of which involve combining MME outputs into single projected climate trajectories. There appear to be two primary reasons that this form of model combination predominates: Simple averaging across models can improve accuracy relative to individual models in historical simulations (38), and modelers perceive policy makers as requiring single projections (as functions of individual GHG emissions scenarios) for use in decision-making (26).

Simple averaging reflects model democracy. Weighted averaging is sometimes performed when it is felt that models can be ranked with respect to relative accuracy in specific fields in historical simulations (even if not overall) (39). Thus, models may be weighted along particular dimensions of policy interest, and the results then averaged.

Expanding on this approach, numerous studies have quantified MME uncertainty and combined model projections by creating multimodel probability distributions across predictions of key variables such as global temperature, and by using them to compute mean trajectories. Bayesian techniques are one approach to doing this-for example, Smith et al. (3) and Sexton et al. (40)_-building, in particular, on the work of Tebaldi et al. (41, 42).

As this type of work has proceeded, climate modelers and scientists and others have recognized some persistent methodological problems in combining model projections $(1,43)$. Regarding the finding that model averages can improve upon the predictions of individual models, Manski $(44,45)$ has shown that this may be the consequence of a mathematical relationship rather than an empirical result. ${ }^{\ddagger}$ Also, model performance with respect to specific fields in historical data has not been demonstrated to imply skill in predicting climate (46), weakening the case for this approach to weighting projections.

Other issues have to do with how relationships among the models, and the selection of model ensemble subsets from the entire set of extant models, affect the informational content and quality of the ensemble results. These topics have been framed in terms of the degrees or levels of model "independence" in ensembles, although the formal meaning of "independence" has not been clear. In particular, one should be wary of conceptualizing independence in statistical terms, in the absence of a probabilistic theory of model development. As noted above, ensembles are typically assembled by convenience, in the sense that they are determined by the availability and interests of modelers. Thus, one cannot reasonably assert that any given ensemble is a random sample drawn from a population of potential models. Nor can one reasonably assert that any given ensemble adequately expresses the full range of structural uncertainty in model projections (24). Indeed, it is not known how well the world's complete set of existing climate models expresses the full range of uncertainty.

While it is inappropriate to conceptualize model independence in statistical terms, there may be good reason to think nonstatistically about dependence across models. Modeling teams communicate with one another, and they may, hence, influence the modeling choices that they collectively make. In fact, some models share parts of their codes (47).

As discussed by Sanderson (1), researchers are actively working on ways to address these problems. However, combining climate model ensemble outputs into single projected trajectories of the future global climate remains a challenging and unresolved problem. In the following sections, we discuss how viewing model structural uncertainty in terms of partial identification facilitates an alternative approach to using MME information in climate policy and decision-making.

\footnotetext{
${ }^{\ddagger}$ Specifically, Jensen's Inequality implies that, when mean square error or another convex loss function is used to measure predictive accuracy, the accuracy of a simple or weighted mean prediction is algebraically better than the corresponding average accuracy of individual model predictions.
} 


\section{Climate Policy under Partial Identification: Cost-Benefit Analysis with the Min-Max and MMR Decision Rules}

The issues with standard approaches to defining and quantifying uncertainties in climate modeling that we have reviewed do not obviate the fact that modeling is essential for developing policies to address the risks of global climate change. What is clearly the case, however, is that addressing uncertainties remains an open, and challenging, problem. Parker (48) observed that a primary rationale for using weighting or probability analysis to combine climate model outputs into single time paths of important variables appears to be modelers' perception that policy makers require uncertainty to be represented in this form to make decisions. In this section, we show that, in fact, this is not the case, and that a partial identification framing of climate modeling opens the way to alternative methods for dealing with uncertainties in decision-making. We might say that these methods allow for single decisions to be made without needing single inputs from the models.

Our departure point is climate model ensemble analysis, so the models discussed in the following sections express partial identification in terms of a discrete set of climate models. As noted earlier, our analysis does not encompass the full uncertainty inherent in numerical climate simulation. By its very nature, decision analysis must explicitly specify possible "states of nature," which list only known unknowns. Our goal is to advance understanding within that paradigm.

Economic Uncertainty in Integrated Assessment Modeling. Although not our focus in this paper, for the sake of context, we begin with a brief discussion of uncertainty in the economic elements of quantitative climate policy analysis, which, as a rule, uses computational energy-economic or integrated assessment (IA) models. (This subject is discussed by Sanstad in ref. 49.) These are, characteristically, long-run (century-scale or more) models of the global economy including the energy system and its role in economic production, and are applied to estimating the costs of policy-induced GHG emissions reductions. IA models incorporate reduced-form representations of the climate (or, more generally, the carbon cycle) and its links to the economy, particularly the climatic effects of anthropogenic GHG emissions and, in turn, their effects on the economy.

The development of IA models has enabled economic analysis of how the global climate and social systems interact with one another, which has played a critical role in the development of policies to reduce GHG emissions (50). IA models are subject to uncertainty not just in their representation of the climate but also in their economic assumptions (50). Examples arise in highly aggregated IA models in which the direct economic costs of climate change and those of GHG abatement are both represented explicitly, so that trade-offs between emissions reduction and reduced climate change-induced economic losses can be assessed. The paradigmatic example is Nordhaus's Dynamic Integrated Climate Economy (DICE) model, the most influential IA model of the last several decades (51).

In DICE and models based on it, the economic losses from climate change are represented in terms of "damage functions" that give the decreases in worldwide output resulting from increases in mean global temperature, as a proportional reduction or in dollar terms. It is recognized that these functions have uncertain theoretical and empirical grounding (52). ${ }^{\S}$

\footnotetext{
${ }^{5}$ Some of this work has focused on improving aggregate damage functions with respect to both aggregate-level evidence and functional forms (63). Moreover, it is also the case that aggregation per se is a major source of this type of uncertainty, and increasing attention is being paid to empirical, statistical estimation of spatially and sectorally disaggregated climate damages $(69,70)$.
}

Another feature of these models is dynamic optimization by a representative decision maker, which entails discounting to quantify the present value of future economic costs and benefits. The appropriate definition and magnitude of the discount rate is a long-standing issue in climate change economics and IA modeling (53-55). Controversy persists, in part, due to the fact that this is not only an empirical question but also a matter of theory and of ethics in regard to intergenerational equity (56).

In addition to these issues, IA model estimates of the long-run costs of large-scale GHG emissions abatement vary considerably, by some measures, up to an order of magnitude (57). As evidenced by comparing these more recent estimates with examples from the 1990s (58), this uncertainty has persisted over several decades of model development.

While improving estimates of the economic impacts of climate change is an active area of research, uncertainty persists. Damage functions in aggregate models continue to be debated, and model-based abatement cost estimates continue to proliferate but are not converging. Because our focus is partial identification of climate models and its implications for decision-making, this paper limits formal analysis of economic uncertainties to sensitivity analysis with respect to the exogenous economic parameters governing abatement and damage costs. However, our decision framework can be readily applied to economic uncertainty.

A Simple Theoretical Framework. In what follows, we consider climate policy from a cost-benefit perspective in the context of partial identification of climate models, in which emissions abatement policy is based on comparing the costs of reducing GHG emissions with the resulting benefit in terms of avoided climate change damages. The key consideration is that the decision maker's knowledge of these benefits is subject to uncertainty arising from the partial identification. We first develop an abstract theoretical framework and then, in the following section, implement it quantitatively using a simple IA model.

Let $B_{t}$ represent the state at time $t$ of a baseline energyeconomic scenario, and let $E_{t}^{B_{t}}$ be the corresponding baseline GHG emissions. Let $A_{t}$ be GHG abatement actions at time $t$ under some climate policy, measured in the same units as emissions, let $C\left(A_{t}\right)$ be their cost, and let $E_{t}^{A_{t}}$ be the resulting net emissions. In what follows, we will slightly abuse terminology by also referring to $A_{t}$ and $E_{t}^{A_{t}}$ as "paths" or "trajectories." We assume that abatement paths are chosen from some space of feasible paths.

Emissions paths are used as inputs to a climate model $M$. We focus on the global mean temperatures projected by $M$ as a function of these paths. Thus, let $T\left(E_{t}^{A_{t}}, M\right)$ be the global mean temperature at time $t$ determined by the GHG trajectory $E_{t}^{A_{t}}$ when it is simulated in the climate model $M$. Then a damage function-as discussed above-can be written as $D\left(T\left(E_{t}^{A_{t}}, M\right)\right)$.

\section{Cost-benefit analysis.}

The min-max decision rule. For an abatement path $A_{t}$ and climate model $M$, denote the associated total (abatement plus damages) cost at time $t$ as $\mathbb{C}\left(A_{t}, M\right) \equiv C\left(A_{t}\right)+D\left(T\left(E_{t}^{A_{t}}, M\right)\right)$. A policy maker seeks to minimize the present value of the cumulative cost over a planning horizon which, as is customary in the climate economics literature, we assume to be infinite. The cost-benefit climate policy problem given a particular climate model $M$ is

$$
\min _{A_{t}} \int_{0}^{\infty} \mathbb{C}\left(A_{t}, M\right) e^{-\delta t} d t,
$$

where $\delta$ is a discount rate. In this approach, the optimal $A_{t}$ is chosen with commitment at time 0 , that is, it is not updated over time as new climate or cost information is obtained. As stated, Eq. $\mathbf{1}$ is a deterministic optimization problem that, under certain technical assumptions regarding the feasible abatement path space and the cost and 
damage functions, has a unique solution. We will assume that such conditions hold for the series of problems we describe.

Reflecting the discussion in Partial Identification and $M M E$ Analysis, let $\mathbf{M}=\left\{M_{1}, \ldots, M_{N}\right\}$ be a model ensemble. We amend the damage and cost function notations to $\mathbb{C}\left(A_{t}, M_{i}\right) \equiv C\left(A_{t}\right)+D\left(T\left(E_{t}^{A_{t}}, M_{i}\right)\right), i=1, \ldots, N$. The hypothetical policy maker now faces the problem of minimizing total cost while taking account of the model uncertainty. One way of approaching this problem is to assign a probability distribution or some other weighting scheme to $\mathbf{M}$ and minimize the expected cost. However, this strategy is unsatisfactory if one lacks a credible basis for assigning a distribution or weights.

Under a partial identification perspective, one alternative way to proceed is to apply the min-max decision rule, which can be characterized colloquially as "preparing for the worst." Here, the "worst" is the model $M_{i}$ that results in the highest total abatement plus damage costs. Given an abatement path $A_{t}$ and associated emissions path $E_{t}^{A_{t}}$, the most costly outcome will correspond to the model that solves the problem

$$
\max _{i} \int_{0}^{\infty} \mathbb{C}\left(A_{t}, M_{i}\right) e^{-\delta t} d t .
$$

This maximum cost will vary across possible abatement paths, and the min-max decision is to select the path that solves

$$
\min _{A_{t}} \max _{i} \int_{0}^{\infty} \mathbb{C}\left(A_{t}, M_{i}\right) e^{-\delta t} d t .
$$

That is, the decision rule chooses the abatement policy that minimizes the total cost of abatement and damages under the most pessimistic assumption regarding the climate model.

The MMR decision rule. Policies chosen using the min-max criterion are conservative by design. An alternative decision rule that is applicable under conditions of partial identification but is less conservative is called MMR, which Manski and Tetenov (59) characterize as seeking uniformly near-optimal solutions to decision problems. For each model $M_{i}$ in the ensemble $\mathbf{M}=$ $\left\{M_{1}, \ldots, M_{N}\right\}$, with $\mathbb{C}\left(A_{t}, M_{i}\right) \equiv C\left(A_{t}\right)+D\left(T\left(E_{t}^{A_{t}}, M_{i}\right)\right)$ the associated time $t$ cost with abatement and emissions paths $A_{t}$ and $E_{t}^{A_{t}}$, respectively, let $A_{t ; M_{i}}^{*}$ be the abatement path that solves the problem

$$
\min _{A_{t}} \int_{0}^{\infty} \mathbb{C}\left(A_{t}, M_{i}\right) e^{-\delta t} d t
$$

and let $\mathbb{C}^{*}\left(A_{t ; M_{i}}^{*}, M_{i}\right)$ be the resulting minimum cost,

$$
\mathbb{C}^{*}\left(A_{t ; M_{i}}^{*}, M_{i}\right) \equiv \min _{A_{t}} \int_{0}^{\infty} \mathbb{C}\left(A_{t}, M_{i}\right) e^{-\delta t} d t
$$

(Note the change in notation: Previously, $\mathbb{C}\left(A_{t}, M_{i}\right)$ was total cost at time $t$; now, $\mathbb{C}^{*}\left(A_{t ; M_{i}}^{*}, M_{i}\right)$ is total discounted cost, i.e., an integral.) Now consider any other feasible trajectory $A_{t}$ and its costnot necessarily optimal-over the entire time horizon with $M_{i}$, $\int_{0}^{\infty} \mathbb{C}\left(A_{t}, M_{i}\right) e^{-\delta t} d t$. The regret $\mathbb{R}\left(A_{t}, M_{i}\right)$ associated with $A_{t}$ when climate model $M_{i}$ is used in determining damages is the difference between its cost and the cost of the optimal policy associated with $M_{i}$,

$$
\mathbb{R}\left(A_{t}, M_{i}\right) \equiv \int_{0}^{\infty} \mathbb{C}\left(A_{t}, M_{i}\right) e^{-\delta t} d t-\mathbb{C}^{*}\left(A_{t ; M_{i}}^{*}, M_{i}\right)
$$

The regret is nonnegative because $\mathbb{C}^{*}\left(A_{t ; M_{i}}^{*}, M_{i}\right)$ is the minimum cost with $M_{i}$. Regret measures the suboptimality of $A_{t}$ when temperature is determined by $M_{i}$. That is, it is the excess cost resulting from this abatement path relative to the minimal cost associated with this model.

To apply the MMR rule, a decision maker first considers each feasible abatement path $A_{t}$ and finds the model version that maximizes regret as defined in Eq. 6, solving the problem

$$
\max _{M_{i}} \mathbb{R}\left(A_{t}, M_{i}\right)=\max _{M_{i}}\left(\int_{0}^{\infty} \mathbb{C}\left(A_{t}, M_{i}\right) e^{-\delta t} d t-\mathbb{C}^{*}\left(A_{t ; M_{i}}^{*}, M_{i}\right)\right) .
$$

The MMR solution is then to find $A_{t}$ to solve the problem

$\min _{A_{t}} \max _{M_{i}} \mathbb{R}\left(A_{t}, M_{i}\right)=\min _{A_{t}} \max _{M_{i}}\left(\int_{0}^{\infty} \mathbb{C}\left(A_{t}, M_{i}\right) e^{-\delta t} d t-\mathbb{C}^{*}\left(A_{t ; M_{i}}^{*}, M_{i}\right)\right)$.

This discussion highlights the fact that, in any application, MMR requires an explicit criterion to measure the differences in social welfare between optimal and suboptimal outcomes. For example, applying the technique to analyze policies that entail exogenously set "caps" on GHG emissions would necessitate measurement of the social cost of exceeding those caps.

\section{A Numerical Example}

In this section, we describe a computational implementation of the MMR framework described above. We present a simple IA model defining an optimal control problem that formalizes the dynamic economic trade-off between emissions abatement and reduced damages from emissions-caused temperature increases. In implementing numerical calculation of the MMR of Eq. 8 in our example, we assume, for simplicity, that the state space of possible real worlds includes only those represented by the six models listed in Table 1. Moreover, we compare only the optimal abatement paths for each of the models. We also contrast the results of applying the MMR criterion with those obtained with the min-max decision rule."

\section{A Simple Integrated Assessment Model.}

Emissions and temperature. In the previous section, we represented the link between carbon emissions, in the form of a trajectory $E_{t}^{A_{t}}$, and projected global mean temperature $T_{t}$ as a functional relationship based on a climate model $M: T_{t} \equiv T\left(E_{t}^{A_{t}}, M\right)$, where $E_{t}^{A_{t}}$ are net emissions at time $t$ when baseline emissions $B_{t}$ are reduced by abatement $A_{t}$, and are used to project temperature using $M$. For quantitative analysis, we now simplify this representation by adapting the approach of Matthews et al. (60) to summarize the longterm relationship between aggregate carbon emissions and global mean temperature. Using a combination of modeling and observational data analysis, these researchers showed that the ratio of temperature increase to cumulative emissions, the "carbon-climate response (CCR)," is roughly constant over decades up to several centuries. We use the CCR to capture the influence of emissions on temperature. (This approach is further discussed in refs. 61, 62.)

Let $\mathbf{E}_{t}^{A_{t}}$ be cumulative emissions between an initial time 0 and time $t$ given a net emissions path $E_{t}^{A_{t}}$,

$$
\mathbf{E}_{t}^{A_{t}}=\int_{0}^{t} E_{s}^{A_{s}} d s
$$

Let $m(M)$ be the CCR associated with a climate model $M$. Then the Matthews et al. (60) approximation of global mean temperature projected by $M$ when it is driven by $E_{t}^{A_{t}}$ is

$$
T_{t}=T\left(E_{t}^{A_{t}}, M\right)=m(M) \int_{0}^{t} E_{s}^{A_{s}} d s=m(M) \mathbf{E}_{t}^{A_{t}} .
$$

Slightly amending our notation from the previous section, we will use the parameter $m$ to characterize the models being compared Different models might, in principle, have the same $m$, but this is

\footnotetext{
TOur model is similar to that of Goulder and Mathai (71), which was adapted to MMR analysis of carbon abatement policy by Cai and Sanstad (11). Rezai and van der Ploeg (12) developed a more complex IA model and applied it to max-min and MMR analysis of climate analysis under uncertainty, using temperature projections from several different IA models.
} 
GFDL-ESM-2G - Geophysical Fluid Dynamics Laboratory ESM version

$2 \mathrm{G}$

BCC-CSM-1 - Beijing Climate Center Climate System Model version 1.1 FIO-ESM - FIO-ESM - First Institute of Oceanography ESM

Had-GEM2-ES - Hadley Global Environmental Model 2 - Earth System

IPSL-CM5A-MR - Institut Pierre Simon Laplace Coupled Model 5A -

Medium Resolution

MIROC-ESM - Model for Interdisciplinary Research on Climate - ESM
1.573

1.864

1.937

2.286

2.361

2.438 not the case for the six we examine here. Thus, we can write the model-projected temperature as $T_{t}=T\left(E_{t}^{A_{t}}, m\right)$.

Costs and damages. We assume that the functions for abatement cost and climate damage at time $t$ are quadratic,

$$
\begin{aligned}
& C\left(A_{t}\right)=\frac{1}{2} \alpha A_{t}^{2} \\
& D\left(T_{t}\right)=\frac{1}{2} \beta\left(m \mathbf{E}_{t}^{A_{t}}\right)^{2}=\frac{1}{2} \beta m^{2}\left(\mathbf{E}_{t}^{A_{t}}\right)^{2} .
\end{aligned}
$$

The quadratic is a convenient and tractable functional form; in the present case, it enables us to solve our optimization model in closed form (see below). The shape of the damage function has been controversial in climate economics. Nordhaus and Moffat (63) conducted a comprehensive survey of empirical damage estimates in the literature and conducted their own estimations; their preferred specification was quadratic.

With $T_{t}=T\left(E_{t}^{A_{t}}, m\right)=m E_{t}^{A_{t}}$, the total cost at time $t$ is

$$
\begin{gathered}
\mathbb{C}\left(A_{t}, m\right) \equiv C\left(A_{t}\right)+D\left(T\left(E_{t}^{A_{t}}, m\right)\right) \\
=\frac{1}{2} \alpha A_{t}^{2}+\frac{1}{2} \beta m^{2}\left(\mathbf{E}_{t}^{A_{t}}\right)^{2} .
\end{gathered}
$$

Optimization. In the abstract framework of $A$ Simple Theoretical Framework, any constraints that might be imposed on the optimizations were implicit. In optimal control problems, the key type of constraint specifies the dynamic relationship between the decision variable and the "state" variable - here $\mathbf{E}_{t}^{A_{t}}$-as an ordinary differential equation. We write the cost minimization problem for a given climate model as

\begin{tabular}{|c|c|c|c|}
\hline \multicolumn{2}{|c|}{$\begin{array}{l}\text { Abatement cost parameter } \alpha \text { (fractional GDP lost per GtC } \\
\text { abated) }\end{array}$} & \multicolumn{2}{|l|}{$\begin{array}{l}\text { Climate damage cost } \\
\text { parameter } \beta \text { (fractional GDP loss } \\
\text { per degrees Celsius increase) }\end{array}$} \\
\hline Low & 0.000078 & Low & 0.014 \\
\hline Median & 0.000125 & Central [Nordhaus and Moffat (63)] & 0.018 \\
\hline High & 0.0002 & High & 0.022 \\
\hline
\end{tabular}

$$
\begin{aligned}
\min _{A_{t}} \int_{0}^{\infty} \frac{1}{2}\left(\alpha A_{t}^{2}+\beta T_{t}^{2}\right) e^{-\delta t} d t \\
\text { subject to } \\
\frac{d}{d t} \mathbf{E}_{t}^{A_{t}}=E_{t}^{A_{t}}=B_{t}-A_{t} \\
T_{t}=m \mathbf{E}_{t}^{A_{t}} \\
\mathbf{E}_{0}^{A_{t}}=\mathbf{E}_{0},
\end{aligned}
$$

Table 2. Values of economic parameters used for calibration where the last expression defines an initial condition for cumulative emissions. Solving Eq. 13 yields the abatement path $A_{t, m}^{*}$ that minimizes the present (discounted) values of abatement costs plus climate damages subject to the relationships among emissions, temperature, and damages. Conceptually, this path makes the optimal trade-off over time between abatement and damages.

\section{Calibration.}

Baseline emissions projection. We created our baseline emissions path using the "Representative Concentration Pathway (RCP) 8.5 " of carbon and other emissions and atmospheric concentrations created by IA modelers circa 2010 (64). It is one of four such scenarios, each corresponding to a different level of global average watts per meter squared in 2100 that was projected in one or more integrated assessment models $(65,66)$. The $8.5 \mathrm{~W} / \mathrm{m}^{2}$ pathway is the highest warming of the RCPs, reflecting a pessimistic scenario regarding the increase in global emissions over the 21st century; it has been used as a baseline in numerous studies.

We used the RCP 8.5 projection of annual carbon emissions (fossil and land use sources) from 2000 to 2500 in gigatons of carbon $(\mathrm{GtC})$, obtained from the RCP repository at the Potsdam Institute for Climate Impact Research (http://www.pik-potsdam.de/ $\sim$ mmalte/rcps/). For a baseline emissions path approximating the projection, we fitted the following functional form to these data:

$$
B_{t}=\left(\theta t+\frac{B_{0}}{\exp (\theta \varphi)}\right) \exp (-\theta(t-\varphi))
$$

where $B_{0}$ is $8 \mathrm{GtC}$, the RCP 8.5 emissions in 2000 . The fitted parameter values are $\theta=0.0123125$ and $\varphi=339.565$. Further details are provided in SI Appendix, section 2.

Temperature projections under structural uncertainty. To calibrate temperature as a function of cumulative emissions for structural uncertainty analysis, we used outputs of six Earth system models (ESMs) from CMIP5 that each projected the effects of RCP 8.5.

We first computed decadal averages of the CMIP5-reported monthly surface air temperature projections from these models under RCP 8.5 for the period 1900-1909 to 2090-2099. Following Matthews et al. (60) as described above, we then calculated decade-by-decade temperature increases over this period relative to 1900-1909 as projected by each ESM, along with the corresponding decade-by-decade increases in cumulative carbon 
Model

Calculations

\begin{tabular}{cccccc}
\hline GFDL & BCC & FIO & Had & IPSL & MIROC \\
0.293 & 0.381 & 0.4029 & 0.509 & 0.531 & 0.554 \\
0.413 & 0.4033 & - & 0.412 & 0.416 & 0.420 \\
& & & & & \\
0.01059 & 0.0004 & 0 & 0.0088 & 0.01274 & 0.01749
\end{tabular}

emissions in RCP 8.5. Extending the notation introduced above, denote the latter quantity as $\mathbf{E}_{\text {Cum }}^{B_{t}}$.

For each ESM, indexed by $i=1, \ldots, 6$, we represented the relationship between temperature and cumulative net emissions as

$$
T_{i}=m_{i} \mathbf{E}_{\text {Cum }}^{B_{t}} .
$$

To estimate the $m_{i}$, we fitted (15) to the decadal average time series for each model. See SI Appendix, section 2 for details.

The six models and fitted values of $m_{i}$ are shown in Table 1 . The range among these estimates is a simple gauge of structural uncertainty. Economic parameters. We derived low, median, and high estimates of the abatement cost parameter $\alpha$ from Dietz and Venmans (67), who summarized global marginal abatement costs as a percent of global economic output as documented in IPCC Fifth Assessment Report. Because these costs were derived from energy-economic and IA model analyses that assumed a 5\% discount rate, we used this value exclusively. For the central value of the damage cost parameter $\beta$, we used Nordhaus and Moffat's (63) central estimate from their preferred (quadratic) specification and estimation of the climate damage function. We also specified low and high values for sensitivity analysis. The values for these parameters are presented in Table 2.

Analytical and Numerical Solutions. Using standard methods, the model is solved in closed form analytically, and the numerical solution is computed using the Mathematica (68) software; details are presented in SI Appendix, section 3. The results are optimal abatement paths $A_{t ; m_{i}}^{*}$ and minimum costs

$$
\begin{gathered}
\mathbb{C}^{*}\left(A_{t ; m_{i}}^{*}, m_{i}\right)=\min _{A_{t}} \int_{0}^{\infty} \mathbb{C}\left(A_{t}, m_{i}\right) e^{-\delta t} d t \\
=\min _{A_{t}} \int_{0}^{\infty} \frac{1}{2}\left(\alpha A_{t}^{2}+\beta T_{t}^{2}\right) e^{-\delta t} d t
\end{gathered}
$$

subject to the constraints in Eq. 13. ${ }^{\#}$ Details are presented in SI Appendix, section 3.

MMR under Structural Uncertainty. We begin with an example of how regrets are calculated, in the case that the First Institute of Oceanography (FIO) model is correct and with the median and central values of $\alpha$ and $\beta$, respectively. Our analysis assumes that the state space of possible real worlds includes only those represented by the six models. That is, it excludes the possibility that all six models are wrong. In principle, the calculations could be done with a larger state space that has more distinct models. However, we reiterate the point made earlier: No finite ensemble can enable analysis of the "unknown unknowns" of climate modeling.

The first row of Table 3 shows $\mathbb{C}^{*}\left(A_{t ; m_{i}}^{*}, m_{i}\right), i=1, \ldots, 6$, the minimum total discounted abatement costs plus climate damages, associated with the optimal trajectory $A_{t ; m_{i}}^{*}$ for each model for the median $\alpha$ and central $\beta$ parameter case. We assign the

\#Under our assumptions, the necessary conditions are also sufficient, and the model will have unique solutions with the parameters we use. indices $i$ in the order in which the models are listed in Table 1, that is, according to ascending values of the CCR parameter.

To explain the second row, we recall the idea of a suboptimal trajectory from The MMR decision rule, here assuming, for the sake of discussion, that FIO $(i=3)$ is the correct model. For legibility, we again amend our previous notation: Let $A_{t, i \neq 3}^{*}$ be the optimal trajectory for one of the models other than FIO and let $E\left(A_{t, i \neq 3}^{*}\right)$ be the net emissions at time $t$ when this trajectory is used to reduce emissions from the baseline $B_{t}$, that is, $E\left(A_{t, i \neq 3}^{*}\right)=B_{t}-A_{t, i \neq 3}^{*}$. We write the resulting cumulative emissions path as

$$
\begin{gathered}
\mathbf{E}_{t}\left(A_{t, i \neq 3}^{*}\right)=\int_{0}^{t} E\left(A_{s, i \neq 3}^{*}\right) d s \\
=\int_{0}^{t}\left(B_{s}-A_{s, i \neq 3}^{*}\right) d s .
\end{gathered}
$$

Now consider using this emissions path to project temperature with the FIO CCR, $m_{3}$,

$$
\begin{aligned}
T_{t} & =T\left(E\left(A_{t, i \neq 3}^{*}\right)\right) \\
& =m_{3} \int_{0}^{t} E\left(A_{s, i \neq 3}^{*}\right) d s \\
& =m_{3} \mathbf{E}_{t}\left(A_{t, i \neq 3}^{*}\right) .
\end{aligned}
$$

That is, $T_{t}=m_{3} E_{t}\left(A_{t, i \neq 3}^{*}\right)$ is the temperature path when the optimal trajectory for a different model is used with the FIO CCR. In turn, we calculate the time $t$ abatement costs associated with $A_{t, i \neq 3}^{*}$ and climate damages associated with $m_{3} E_{t}\left(A_{t, i \neq 3}^{*}\right)$,

$$
\begin{gathered}
\mathbb{C}\left(A_{t, i \neq 3}^{*}, m_{3}\right)=C\left(A_{t, i \neq 3}^{*}\right)+D\left(T\left(E\left(A_{t, i \neq 3}^{*}\right)\right)\right) \\
=\frac{1}{2} \alpha\left(A_{t, i \neq 3}^{*}\right)^{2}+\frac{1}{2} \beta m_{3}^{2}\left(\mathbf{E}_{t}\left(A_{t, i \neq 3}^{*}\right)\right)^{2} .
\end{gathered}
$$

The present value of these costs is then

$$
\begin{gathered}
\mathbb{C}_{P V}\left(A_{t, i \neq 3}^{*}, m_{3}\right)=\int_{0}^{\infty}\left(C\left(A_{t, i \neq 3}^{*}\right)+D\left(T\left(E\left(A_{t, i \neq 3}^{*}\right)\right)\right)\right) e^{-\delta t} d t \\
=\int_{0}^{\infty}\left(\frac{1}{2} \alpha\left(A_{t, i \neq 3}^{*}\right)^{2}+\frac{1}{2} \beta m_{3}^{2}\left(\mathbf{E}_{t}\left(A_{t, i \neq 3}^{*}\right)\right)^{2}\right) e^{-\delta t} d t .
\end{gathered}
$$

Eq. 20 displays the suboptimality we have referred to: It is a cost, but not the optimal cost, using the FIO model to project temperature. These suboptimal costs for the models $i=1,2,4,5$, and 6 comprise the second row of Table 3 .

The last row of Table 3 presents the associated regrets, the differences between the suboptimal costs and the optimal (minimum) cost with the FIO model,

$$
\mathbb{R}\left(A_{t, i \neq 3}^{*}, m_{3}\right)=\mathbb{C}_{P V}\left(A_{t, i \neq 3}^{*}, m_{3}\right)-\mathbb{C}^{*}\left(A_{t, m_{3}}^{*}, m_{3}\right),
$$

for $i=1,2,4,5$, and 6. As discussed in The MMR decision rule, these are the post hoc losses when a decision maker implements 
Table 4. Values of regret across six ESMs, median $\alpha$ and central $\beta$, including MMR solution (in bold italics)

\begin{tabular}{lllllllr} 
& \multicolumn{6}{c}{ Incorrect model } & \\
\cline { 2 - 6 } Correct Model & \multicolumn{1}{c}{ GFDL } & \multicolumn{1}{c}{ BCC } & FIO & Had & IPSL & MIROC & Row maxima \\
\hline GFDL & 0 & 0.00608 & 0.00939 & 0.03436 & 0.04137 & 0.04914 & 0.04914 \\
BCC & 0.00669 & 0 & 0.00039 & 0.01266 & 0.01726 & 0.02266 & 0.02266 \\
FIO & 0.01059 & 0.0004 & 0 & 0.0088 & 0.01274 & 0.01749 & 0.01749 \\
Had & 0.04357 & 0.01459 & 0.0099 & 0 & 0.00041 & 0.00166 & 0.04357 \\
IPSL & 0.05379 & 0.0204 & 0.0147 & 0.00042 & 0 & 0.00043 & 0.05379 \\
MIROC & 0.06558 & 0.02748 & 0.02071 & 0.00175 & 0.00044 & 0 & 0.06558 \\
\hline
\end{tabular}

the optimal abatement policy assuming that one of the models other than FIO is correct, but FIO is, in fact, the correct model.

The full set of regrets across the six models for the median $\alpha$ and central $\beta$ case is shown in Table 4,

$$
\mathbb{R}\left(A_{t, i \neq j}^{*}, m_{j}\right)=\mathbb{C}_{P V}\left(A_{t, i \neq j}^{*}, m_{j}\right)-\mathbb{C}^{*}\left(A_{t, m_{j}}^{*}, m_{j}\right),
$$

$i, j=1, \ldots, 6$. The rows are regrets with respect to the models listed at the left, with the FIO row values equal to those in the last line of Table 3. For example, the number in the second row, fourth column (0.01266) is the regret from using the Hadley (Had) model to perform the optimization when the correct model is the Beijing Climate Center (BCC). The MMR solution across the entire table is shown in bold italics in Table 4, and occurs when FIO is the correct model and the Model for Interdisciplinary Research on Climate (MIROC) is used instead for the optimization.

Note that the values in each row form a "U" shape with respect to the zero value for the correct model for the row. In the rows with the models having the three lowest CCRs-Geophysical Fluid Dynamics Laboratory (GFDL), BCC, and FIO-the maximum regret occurs with the MIROC model, while in the rows with the models having the three highest CCRs-Had, Institut Pierre Simon Laplace (IPSL), and MIROC - it occurs with the GFDL. This is intuitive given the relative magnitudes of the various CCRs.

This calculation of the MMR solution assumes that the action space (that is, the set of feasible abatement paths) includes only the six abatement paths that are optimal under one of the models. Thus, it takes the action space to be the set of "best response" paths. More generally, society could consider abatement paths that are not optimal under any of the six models. Even if the state space comprises only the six models, it is possible that the MMR path will be one that is not optimal in any state. Thus, Table 4 gives a constrained MMR solution. Finding the unconstrained MMR abatement path would be a much more complex computational problem.

To explore the sensitivity of the MMR to the economic parameters, we calculated nine tables for the nine $\alpha, \beta$ pairs. Table 5 summarizes the results, showing the MMR for each combination of $\alpha$ and $\beta$. For all these parameter pairs, the MMR occurs with FIO as the model selected for optimization and MIROC as the incorrect model.

It is interesting to see that the MMR is increasing in $\beta$ for each value of $\alpha$; that is, the greater the sensitivity of climate damages to cumulative carbon, the greater the regret for using an incorrect ESM. The MMR is also increasing in $\alpha$ for the central and high values of $\beta$, but not for the low value.

A sensitivity analysis of the model with respect to the economic parameters is presented in SI Appendix, section 3; its results and the data in Table 4 allow us to compare the MMR and min-max decision rule outcomes. The sensitivity analysis shows that the MIROC model yields the highest cost optimal solution for all $\alpha, \beta$ pairs-that is, if MIROC correctly embodies the
CCR, the outcome is the worst using our optimization model. It is natural to ask, What will the cost turn out to be if MIROC is correct, but another model is chosen? The answer is, of course, the set of regrets associated with MIROC. Comparing the MIROC and FIO rows in Table 4, we see that the MIROC regrets are, in some cases, higher and, in some cases, lower than those for FIO. But its maximum regret (0.06558, when GFDL is chosen but MIROC is correct) is greater than FIO's maximum regret $(0.01749)$. The difference between the two can be interpreted as the potential cost of using the min-max rather than the MMR decision rule with this optimization model.

\section{Conclusion}

Computational climate modeling is a challenging endeavor. Over decades, the efforts of many modelers around the world have yielded steady progress in model development. The models enable policy makers to analyze the long-range consequences of societal decisions about energy production and use. However, the complexities of the climate system and computational difficulties generate structural uncertainty. A consequence is that a number of distinct models have been developed over time and are currently in use.

Taking account of this uncertainty in IA modeling and other policy applications has, with few exceptions, relied on climate model weighting of one kind or another, including Bayesian probabilistic analysis. The shortcomings of these weighting schemes are known and well documented in the technical literature. As an alternative, we have proposed a partial identification framing and cost-benefit analysis of climate policy using the MMR criterion. We have provided a theoretical treatment of MMR decision-making under structural uncertainty, and a simple numerical IA example. The MMR criterion offers a rationally grounded way to deal with structural uncertainty, and our results establish that the partial identification approach is a promising avenue for further development of computational modeling for climate policy.

There are several ways this work could be extended. MMR analysis of joint parametric and structural climate model uncertainty could be conducted by explicitly distinguishing the two in the link between cumulative emissions and temperature. Of particular interest is MMR analysis of the types of economic uncertainties we discussed, both in their own right and jointly

Table 5. Values of MMR among six climate models across economic parameters (from the FIO/MIROC model pair in each case)

\begin{tabular}{lccc} 
& \multicolumn{3}{c}{ Value of $\beta$} \\
\cline { 2 - 4 } Value of $\alpha$ & Low & Central & High \\
\hline & & & \\
Low & 0.01300 & 0.01538 & 0.01719 \\
Median & 0.01387 & 0.01749 & 0.02053 \\
High & 0.01312 & 0.01763 & 0.02179 \\
\hline
\end{tabular}


with climate model uncertainty. Such research could greatly enhance the capacity of decision makers to address this most challenging of environmental problems.

Data Availability. All study data are included in the article and SI Appendix.

1. B. M. Sanderson, "Uncertainty quantification in multi-model ensembles" in Oxford Research Encyclopedia of Climate Science (Oxford University Press, 2018).

2. R. Knutti, The end of model democracy? Clim. Change 102, 395-404 (2010).

3. R. L. Smith, C. Tebaldi, D. Nychka, L. O. Mearns, Bayesian modeling of uncertainty in ensembles of climate models. J. Am. Stat. Assoc. 104, 97-116 (2009).

4. A. Wald, Statistical Decision Functions (Wiley, New York, 1950).

5. L. J. Savage, The theory of statistical decision. J. Am. Stat. Assoc. 46, 55-67 (1951).

6. C. Manski, Search profiling with partial knowledge of deterrence. Econ. J. (Lond.) 116, F385-F401 (2006)

7. C. F. Manski, Vaccination with partial knowledge of external effectiveness. Proc. Natl. Acad. Sci. U.S.A. 107, 3953-3960 (2010).

8. R. Loulou, A. Kanudia, Minimax regret strategies for greenhouse gas abatement: Methodology and application. Oper. Res. Lett. 25, 219-230 (1999).

9. T. Iverson, Communicating trade-offs amid controversial science: Decision support for climate policy. Ecol. Econ. 77, 74-90 (2012).

10. D. Anthoff, R. Tol, Climate policy under fat-tailed risk: An application of FUND. Ann. Oper. Res. 220, 223-237 (2014)

11. Y. Cai, A. H. Sanstad, Model uncertainty and energy technology policy: The example of induced technical change. Comput. Oper. Res. 66, 362-373 (2016).

12. A. Rezai, F. van der Ploeg, Climate policies under climate model uncertainty: Max-min and min-max regret. Energy Econ. 68, 4-16 (2017).

13. Y. Cai, Computational methods in environmental and resource economics. Annu. Rev. Resour. Econ. 11, 59-82 (2019).

14. T. Koopmans, Identification problems in economic model construction. Econometrica 17, 125-144 (1949).

15. C. Manski, Partial Identification of Probability Distributions (Springer-Verlag, New York, 2003).

16. C. Manski, Identification for Prediction and Decision (Harvard University Press, Cambridge, MA, 2007).

17. E. Tamer, Partial identification in econometrics. Annu. Rev. Econ. 2, 167-195 (2010).

18. F. Molinari, "Microeconomics with partial identification" in Handbook of Econometrics, S. N. Durlauf, L. P. Hansen, J. J. Heckman, R. L. Matzkin, Eds. (North-Holland, 2020), pp. 355-486.

19. K. McGuffie, A. Henderson-Sellers, A Climate Modelling Primer (John Wiley, Chichester, UK, ed. 3, 2005).

20. D. Bader et al; CCSP, Climate Models: An Assessment of Strengths and Limitations. A Report by the U. S. Climate Change Science Program and the Subcommittee on Global Change Research (Department of Energy, Washington, DC, 2008).

21. J. C. McWilliams, Irreducible imprecision in atmospheric and oceanic simulations. Proc. Natl. Acad. Sci. U.S.A. 104, 8709-8713 (2007).

22. T. N. Palmer et al., Representing model uncertainty in weather and climate predic tion. Annu. Rev. Earth Planet. Sci. 33, 163-193 (2005).

23. D. A. Stainforth, M. R. Allen, E. R. Tredger, L. A. Smith, Confidence, uncertainty and decision-support relevance in climate predictions. Philos. Trans. A Math. Phys. Eng. Sci. 365, 2145-2161 (2007)

24. R. Knutti, R. Furrer, C. Tebaldi, J. Cermak, G. A. Meehl, Challenges in combining projections from multiple climate models. J. Clim. 23, 2739-2758 (2010)

25. W. S. Parker, Ensemble modeling, uncertainty and robust predictions. Wiley Interdiscip. Rev. Clim. Change 4, 213-223 (2013).

26. W. S. Parker, Understanding pluralism in climate modeling. Found. Sci. 11, 349-368 (2006)

27. S. J. Lambert, G. J. Boer, Atmosphere-ocean heat fluxes and stresses in general circulation models. Atmos. Ocean 27, 692-715 (1989).

28. G. A. Meehl, G. J. Boer, C. Covey, M. Latif, R. J. Stouffer, The Coupled Model Intercomparison Project. Bull. Am. Meteorol. Soc. 81, 313-318 (2000).

29. G. A. Meehl, C. Covey, B. McAvaney, M. Latif, R. J. Stouffer, Overview of the Coupled Model Intercomparison Project. Bull. Am. Meteorol. Soc. 86, 89-93 (2005).

30. K. E. Taylor, R. J. Stouffer, G. A. Meehl, An overview of CMIP5 and the experimental design. Bull. Am. Meteorol. Soc. 93, 485-498 (2012).

31. P. J. Gleckler, K. E. Taylor, C. Doutriaux, Performance metrics for climate models. J. Geophys. Res. 113, D06104 (2008)

32. M. Collins et al., Climate model errors, feedbacks, and forcings: A comparison of perturbed physics and multi-model ensembles. Clim. Dyn. 36, 1737-1766 (2011).

33. C. Wang, L. Zhang, S.-K. Lee, L. Wu, C. R. Mechoso, A global perspective on CMIP5 climate model biases. Nat. Clim. Chang. 4, 201-205 (2014).

34. A. Gainusa-Bogdan, F. Hourdin, A. K. Traore, P. Braconnot, Omens of coupled model biases in the CMIP5 AMIP simulations. Clim. Dyn. 51, 2927-2941 (2018).

35. B. J. Soden, W. D. Collins, D. R. Feldman, Reducing uncertainties in climate models Science 361, 326-327 (2018).

36. World Climate Research Programme, Overview CMIP6 experimental design and organization. https://www.wcrp-climate.org/wgcm-cmip/wgcm-cmip6. Accessed 29 June 2019.

37. G. Abramowitz et al., Model dependence in multi-model climate ensembles: Weighting sub-selection and out-of-sample testing. Earth Syst. Dynam. 10, 91-105 (2019).

38. T. Reichler, J. Kim, How well do coupled models simulate today's climate? Bull. Am. Meteorol. Soc. 89, 303-311 (2008).
ACKNOWLEDGMENTS. A.H.S. thanks the NSF and the Center for Robust Decision-Making on Climate and Energy Policy at the University of Chicago for supporting previous research that contributed to this work. We thank the research team of Professor Elizabeth Moyer (Department of Geosciences, University of Chicago) for providing CMIP5 model output, and Valentyn Litvin (Department of Economics, Northwestern University) for his technical review. We have benefitted greatly from the comments of an anonymous reviewer.

39. F. Giorgi, L. O. Mearns, Probability of regional climate change based on the Reliability Ensemble Averaging (REA) method. Geophys. Res. Lett. 30, 1629 (2003).

40. D. M. H. Sexton, J. M. Murphy, M. Collins, M. J. Webb, Multivariate probabilistic projections using imperfect climate models part I: Outline of methodology. Clim. Dyn. 38, 2513-2542 (2012)

41. C. Tebaldi, L. Mearns, D. Nychka, R. Smith, Regional probabilities of precipitation change: A Bayesian analysis of multimodel simulations. Geophys. Res. Lett. 31, L24213 (2004)

42. C. Tebaldi, R. L. Smith, D. Nychka, L. O. Mearns, Quantifying uncertainty in projections of regional climate change: A Bayesian approach to the analysis of multimodel ensembles. J. Clim. 18, 1524-1540 (2005).

43. C. Tebaldi, R. Knutti, The use of the multi-model ensemble in probabilistic climate projections. Philos. Trans. A Math. Phys. Eng. Sci. 365, 2053-2075 (2007).

44. C. Manski, "Interpreting and combining heterogeneous survey forecasts" in Oxford Handbook on Economic Forecasting, M. Clements, D. Hendry, Eds. (Oxford University Press, 2011), pp. 457-472.

45. C. Manski, Interpreting point predictions: Some logical issues. Found. Trends Accounting 10, 238-261 (2016)

46. G. Flato, J. Marotzke, "Evaluation of climate models" in Climate Change 2013: The Physical Science Basis. Contribution of Working Group I to the Fifth Assessment Report of the Intergovernmental Panel on Climate Change, T. F. Stocker et al., Eds. (Cambridge University Press, 2013), pp. 741-866.

47. R. Knutti, D. Masson, A. Gettelman, Climate model genealogy: Generation CMIP5 and how we got there. Geophys. Res. Lett. 40, 1194-1199 (2013).

48. W. S. Parker, Whose probabilities? Predicting climate change with ensembles of models. Philos. Sci. 77, 985-997 (2010).

49. A. H. Sanstad, Energy-economic policy modeling. AIP Conf. Proc. 1924, 020011 (2018).

50. J. Weyant, Some contributions of integrated assessment models of global climate change. Rev. Env. Econ. Policy 11, 115-137 (2017).

51. W. D. Nordhaus, Climate change: The ultimate challenge for economics. Am. Econ. Rev. 109, 1991-2014 (2019).

52. R. Pindyck, Climate change policy: What do the models tell us? J. Econ. Lit. 51, 860-872 (2013)

53. F. Ackerman, S. J. DeCanio, R. B. Howarth, K. Sheeran, Limitations of integrated as sessment models of climate change. Clim. Change 95, 297-315 (2009).

54. D. A. Weisbach, C. R. Sunstein, Climate change and discounting the future: A guide for the perplexed. Yale Law Policy Rev. 27, 433-457 (2009).

55. R. Pindyck, The use and misuse of models for climate policy. Rev. Environ. Econ. Policy 11, 100-114 (2017).

56. P. Dasgupta, Discounting climate change. J. Risk Uncertainty 37, 141-169 (2008).

57. L. Clarke, K. Jiang, "Assessing transformation pathways" in Climate Change 2014 Mitigation of Climate Change. Contribution of Working Group III to the Fifth As sessment Report of the Intergovernmental Panel on Climate Change, O. Edenhofe et al., Eds. (Cambridge University Press, Cambridge, UK, 2014), pp. 413-510.

58. C. Fischer, R. D. Morgenstern, Carbon abatement costs: Why the wide ranges of estimates? Energy J. 27, 73-86 (2006)

59. C. F. Manski, A. Tetenov, Sufficient trial size to inform clinical practice. Proc. Natl. Acad. Sci. U.S.A. 113, 10518-10523 (2016)

60. H. D. Matthews, N. P. Gillett, P. A. Stott, K. Zickfeld, The proportionality of global warming to cumulative carbon emissions. Nature 459, 829-832 (2009).

61. National Academies of Sciences, Engineering, and Medicine, Assessment of Approaches to Updating the Social Cost of Carbon: Phase 1 Report on a Near-Term Update. Committee on Assessing Approaches to Updating the Social Cost of Carbon, Board on Environmental Change and Society (The National Academies Press, Washington, DC, 2016).

62. W. R. Cline, Transient climate response to cumulative emissions (TCRE) as a reducedform climate model. https://secureservercdn.net/50.62.174.113/g80.5df.myftpupload. com/wp-content/uploads/2020/05/EIIWP20-02.pdf. Accessed 23 November 2020

63. W. D. Nordhaus, A. Moffat, A survey of global impacts of climate change: Replication, survey methods, and a statistical analysis. NBER [Preprint] (2017). https://www.nber. org/papers/w23646 (Accessed 19 September 2019)

64. K. Riahi et al., RCP 8.5 - A scenario of comparatively high greenhouse gas emissions. Clim. Change 109, 33-57 (2011).

65. R. H. Moss et al., The next generation of scenarios for climate change research and assessment. Nature 463, 747-756 (2010).

66. M. Meinshausen et al., The RCP greenhouse gas concentrations and their extensions from 1765 to 2300. Clim. Change 109, 213-241 (2011).

67. S. Dietz, F. Venmans, Cumulative carbon emissions and economic policy: In search of general principles. J. Environ. Econ. Manage. 96, 108-129 (2019)

68. Wolfram Research, Inc., Mathematica 12 (Wolfram Research, Champaign, IL, 2020)

69. S. Hsiang et al., Estimating economic damage from climate change in the United States. Science 356, 1362-1369 (2017)

70. M. Auffhammer, Quantifying economic damages from climate change. J. Econ. Perspect. 32, 33-52 (2018).

71. L. H. Goulder, K. Mathai, Optimal $\mathrm{CO}_{2}$ abatement in the presence of induced technical change. J. Environ. Econ. Manage. 39, 1-38 (2000). 